\title{
Alteraciones neuropsicológicas en el alcoholismo: una revisión sobre la afectación de la memoria y las funciones ejecutivas.
}

\author{
Natalia Landa* \\ Javier Fernández-Montalvo** \\ JaVier TiRAPU UstarRoz * * \\ * Psicóloga Clínica. Fundación Proyecto Hombre de Navarra. \\ * Departamento de Psicología y Pedagogía. Universidad Pública de Navarra. \\ *** Servicio de Neuropsicología. Clínica Ubarmin. Pamplona.
}

\section{RESUMEN}

El estudio de los déficit neuropsicológicos en los sujetos alcohólicos ha cobrado una especial relevancia en las últimas décadas. Esto se debe fundamentalmente a sus implicaciones en aspectos tan importantes como son el pronóstico terapéutico y el diseño de estrategias adecuadas de intervención. Este artículo presenta una revisión de los principales estudios sobre la afectación de la memoria y de las funciones ejecutivas en alcohólicos. Los resultados, aunque con ciertas discrepancias, sugieren la existencia de una alteración en la capacidad mnésica, que afecta sobre todo a la memoria de trabajo y a estrategias de aprendizaje. Asimismo se constata la presencia de un déficit en las funciones ejecutivas en los sujetos alcohólicos. Por otro lado, se valora la posibilidad de que esté déficit en las funciones ejecutivas actúe como un factor de vulnerabilidad a desarrollar alcoholismo.

Palabras clave: Alcoholismo. Déficit neuropsicológicos. Memoria de trabajo. Funciones ejecutivas. Vulnerabilidad.

\begin{abstract}
Research into neuropsychological deficit in alcoholics has acquired a special prominence in the last decade. This is due to its importance for both the therapeutic outcome and the therapeutic design. A review of the main studies on memory impairments and on executive cognitive functioning in alcoholics is presented in this article. Results show a disturbance in the memory, especially in the working memory and in the learning strategies, although there are some discrepancies. In addition, data supporting the existence of a dysexecutive syndrome in alcoholics have been reported. Lastly, the influence of the executive cognitive function as a vulnerability factor in developing alcoholism is analysed.
\end{abstract}

Key-words: Alcoholism. Neuropsychological impairment. Working memory. Executive cognitive functioning. Vulnerability.

\section{INTRODUCCIÓN}

E n la pasada década la investigación sobre el comportamiento humano ha centrado su atención sobre un nuevo nivel de análisis: la relación entre la conducta, los procesos mentales y el cerebro. En este contexto, la neuropsicología ha irrumpido con fuerza. Parece evidente que cualquier conducta humana debe ser estudiada en relación con su substrato neuroanatómico. Esta eclosión de la neuropsicología se explica por la concurrencia de varios factores: a) el acercamiento entre la neuropsicología clínica (que estudia las alteraciones en pacientes afectados por una lesión cerebral) y la psicología cognitiva (que ofrece modelos del procesamiento de la información en cerebros sanos); b) el gran desarrollo de las técnicas de neuroimagen estructural y funcional; c) el interés de algunos investigadores por encontrar el substrato cerebral de algunas patologías consideradas hasta ahora como "funcionales"; y d) la necesidad de cuantificar los datos "blandos" (excesivamente mentalistas) de la psicología.

En este sentido, son numerosas las investigaciones que tratan de encontrar las alteraciones neuropsicológicas presentes en diferentes trastornos mentales, incluso en aquellos considerados tradicionalmente como "funcionales", esto es, sin una base neurobiológica que los explique. Este es el caso, por ejemplo, de los estu- 
dios sobre la anorexia (Fassino, Piero, Daga, Leombruni, Mortara y Rovera, 2002), el trastorno obsesivo compulsivo (Head, Bolton y Hymas, 1989) o el trastorno antisocial de la personalidad (Deckel, Hesselbrock y Basuer, 1996).

Por lo que se refiere a las conductas adictivas los estudios neuropsicológicos más recientes se centran en dos aspectos. Por un lado, se ha intentado encontrar el sustrato neurobiológico de los trastornos adictivos. Se trata de estudios basados en modelos experimentales de laboratorio y en técnicas de neuroimagen funcional, que han descrito el denominado "Sistema de Recompensa Cerebral" ligado al sistema dopaminérgico (Caballero, 2000; Fernández-Espejo, 2002). Por otro lado, se ha estudiado el tipo concreto de habilidades cognitivas preservadas y afectadas en sujetos adictos. Estos últimos estudios intentan establecer un perfil neurocognitivo de las conductas adictivas.

En el caso concreto del alcoholismo, el estudio de los déficit cognitivos asociados al consumo de alcohol cuenta con una larga tradición. No se debe olvidar en este sentido, que ya a finales del siglo XIX se describió el síndrome de Wernicke-Korsakoff asociado al consumo crónico de alcohol y que cursa, básicamente, con un deterioro grave de la memoria. Sin embargo, es en los últimos años cuando se han desarrollado los principales estudios sobre estos déficit cognitivos, en la medida que han aparecido nuevos modelos explicativos sobre el funcionamiento cerebral, así como técnicas más precisas para su estudio.

Hay que señalar que la bibliografía existente sobre los déficit neuropsicológicos en el alcoholismo se agrupa principalmente en torno a tres hipótesis explicativas sobre el patrón de deterioro existente. Por un lado, algunos investigadores afirman que los datos avalan la hipótesis de la vulnerabilidad del hemisferio derecho y señalan que este hemisferio es más sensible al consumo de alcohol (Bertera y Parsons, 1978). Por otro lado, otros autores sostienen que la neurotoxicidad del alcohol afecta a estructuras cortico-subcorticales, mientras que la deficiencia de tiamina afecta fundamentalmente a las regiones cerebrales basales (Iruarrizaga, MiguelTobal, Cano, 2001). Por último, la hipótesis de la vulnerabilidad diferencial del lóbulo frontal mantiene que esta región cerebral, por sus especiales características, es más sensible a los efectos tóxicos del alcohol (Nöel, Van der Linden, Schmidt, Sferraza, Hanak, Le Bon, De Mol, Kornreich, Pelc y Verbank, 2001; Ratti, Soragna, Sibilla, Giardini, Albergati, Savoldi y Bo, 1999; Tarter, 1975-1976).

Desde una perspectiva terapéutica, el estudio neuropsicológico de los pacientes alcohólicos cobra una especial relevancia. El perfil cognitivo de estos pacientes puede constituir un factor pronóstico importante y señalar las líneas terapéuticas más adecuadas. Por otra parte, los estudios más recientes tratan de determinar si estas alteraciones neuropsicológicas son causa o consecuencia del consumo de alcohol y, en el caso de que estos déficit fueran previos (factor de vulnerabilidad), qué implicaciones tienen en el diseño de programas de prevención del trastorno.

El objetivo de este artículo es realizar una revisión sobre los trabajos más recientes que evalúan los déficit de memoria y las alteraciones en las funciones ejecutivas de los sujetos con problemas de alcohol o con vulnerabilidad a padecerlos. Ambas funciones cognitivas son las más estudiadas en la población alcohólica y las más vulnerables al efecto tóxico de esta sustancia. Además, los déficit mnésicos y las alteraciones en las funciones ejecutivas tienen un efecto más devastador en el pronóstico y en la evolución de estos pacientes.

\section{DÉFICIT DE MEMORIA}

El estudio de la memoria ocupa un lugar significativo dentro del estudio de los déficit cognitivos en sujetos alcohólicos (Oscar-Berman y Ellis, 1987). Desde que en 1890 Sergei Korsakoff describiera por primera vez el síndrome amnésico que lleva su nombre, han sido muchos los estudios que tratan de determinar la afectación de esta función cognitiva en los alcohólicos. Este síndrome se caracteriza por un conjunto de alteraciones emocionales y cognitivas entre las que destaca una marcada pérdida de memoria junto con la incapacidad para realizar nuevos aprendizajes. Sin embargo, los estudios al respecto son contradictorios; aún no hay evidencia de la presencia de un deterioro gradual de la memoria previo a la fase de demencia, e incluso algunas investigaciones apuntan lo contrario (Krabbendam, Visser, Derix, Verhey, Hofman, Verhoe, Tuinier y Jolles, 2000).

La incongruencia de estos resultados se explica, en parte, por la complejidad de la memoria humana. Aunque en el modelo clásico (Squire, 1975; Walker, 1976) se consideraba la memoria como una entidad unitaria y homogénea para cuya clasificación se tenía en cuenta tan solo el factor "tiempo de retención" (memoria sensorial, memoria a corto plazo y memoria a largo plazo), esta concepción está en la actualidad muy cuestionada. Se considera que la memoria es una función cognitiva compleja que incluye varios subsistemas, que se sustentan en substratos neuroanatómicos diferenciados y cuyo patrón de deterioro es heterogéneo (Tulving, 1993). La taxonomía de la memoria con mayor aceptación en la actualidad se muestra en la figura 1.

Como puede observarse, se establece una distinción entre la memoria declarativa y la memoria procedimental. En la primera, el acceso a los recuerdos se realiza de forma consciente. Tulving (1972) establece, dentro de este tipo de memoria, una distinción entre la 


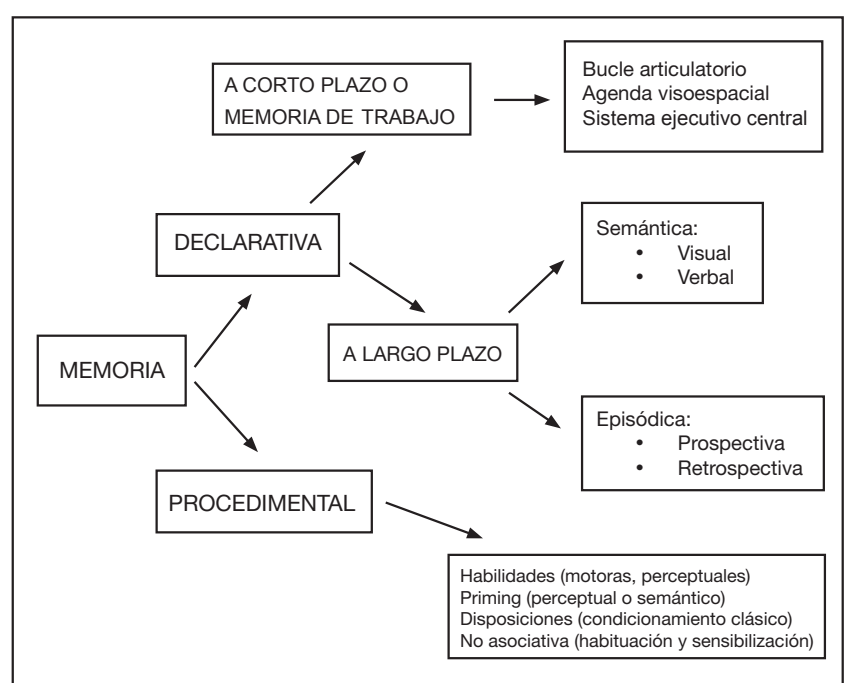

Figura 1. Taxonomía de la memoria (Tirapu, Casi y Ugarteburu ,1997)

memoria episódica, que es de naturaleza autobiográfica y recoge eventos que se almacenan en un marco espacio-temporal de referencia, y la memoria semántica que comprende el conocimiento de hechos de información general. Por su parte, la memoria procedimental incluye mecanismos no conscientes de recuperación y se denomina, por ello, memoria implícita. Por tanto, se describe como un sistema de acción que opera a través de la conducta, sin que precise de mediación cognitiva o experiencia consciente (Squire y Butters,1992).

Por otra parte, la memoria de trabajo, descrita por Baddeley-Hitch (1974), esta compuesta por tres subsistemas: el bucle articulatorio (que permite el mantenimiento activo de información verbal), la agenda visoespacial (que permite la creación, mantenimiento y manipulación de imágenes visuales) y el sistema ejecutivo central (sistema atencional por medio del cual se llevan a cabo las tareas cognitivas en las que interviene la memoria de trabajo y que regula las operaciones de selección de estrategias y de control). Este tipo de memoria es clave a la hora de realizar nuevos aprendizajes y, como se señala posteriormente, es probablemente la más afectada en sujetos alcohólicos.

Respecto al sustrato neuroanatómico de las diferentes memorias, se ha encontrado que la memoria de trabajo (operativa o a corto plazo) se sustenta en el neocórtex y, más en concreto, en la zona de transición parieto-occipito-temporal y el lóbulo frontal medio y anterior (Gómez Bosque,1992). Por otro lado, la memoria declarativa a largo plazo se localiza en estructuras que forman el complejo hipocampo-corteza entorrinalnúcleo amigdalino (sistema temporal medial) y en el complejo tubérculo-mamilar-núcleo dorsomedial del tálamo (Zola, 1997). Por último, la memoria procedimental estaría relacionada con el siguiente locus anatómico: córtex premotor y motor, ganglios basales, núcleo caudado, cerebelo, amígdala y vías reflejas (Vallar, 1999).

Por tanto, debido a la complejidad de esta función la pregunta no debería ser si el consumo de alcohol afecta a la memoria sino qué subsistemas de la memoria se encuentran alterados en los sujetos alcohólicos. Aunque algunos estudios apuntan que el déficit es relativamente generalizado, la mayoría llevan a cabo evaluaciones parciales de la memoria, por lo que no puede concluirse la existencia de un deterioro global. Es probable que la enorme variabilidad de resultados que presentan los diferentes estudios dependa, al menos en parte, del tipo de prueba aplicada y del subsistema de memoria evaluado. Un resumen de los resultados obtenidos hasta el momento se muestra en la tabla 1.

TABLA 1. Estudios sobre la afectación de la memoria en sujetos alcohólicos

\begin{tabular}{|c|c|c|c|}
\hline \multirow[b]{2}{*}{ Autores y año } & \multirow[b]{2}{*}{ Tests empleados } & \multicolumn{2}{|c|}{ Hallazgos } \\
\hline & & Déficit & $\begin{array}{l}\text { Habilidades conserva- } \\
\text { das }\end{array}$ \\
\hline Kapur y Butters, 1977 & Aprendizaje de pares asociados & - Memoria visual & \\
\hline Query y Berger, 1980 & Rey Auditory Verbal Learning Test & - $\quad$ Memoria de trabajo & \\
\hline Leber y col., 1981 & $\begin{array}{l}\text { Escala Revisada de Memoria Wechsler } \\
\text { (versión de Rusell) }\end{array}$ & - $\quad$ Memoria visual & \\
\hline Tarquini y Masullo, 1981 & Rey Auditory Verbal Learning Test & - Memoria de trabajo & \\
\hline Brandt y col., 1983 & Aprendizaje de pares asociados & - $\quad$ Memoria de trabajo & - Memoria visual \\
\hline Acker y col. 1984 & $\begin{array}{l}\text { Escala Revisada de Memoria Wechsler } \\
\text { (versión de Rusell) }\end{array}$ & $\begin{array}{ll}\text { - } & \text { Memoria general } \\
\text { - } & \text { Aención y concen- } \\
\text { - } & \text { tración } \\
\text { - } & \text { Memoria visual } \\
\end{array}$ & - Memoria verbal \\
\hline Becker y Jaffe, 1984 & Aprendizaje de pares asociados & - $\quad$ Memoria de trabajo & \\
\hline Riege y col., 1984 & $\begin{array}{l}\text { Escala Revisada de Memoria Wechsler } \\
\text { (versión de Rusell) }\end{array}$ & & - Memoria visual \\
\hline
\end{tabular}




\begin{tabular}{|c|c|c|c|}
\hline \multirow[b]{2}{*}{ Autores y año } & \multirow[b]{2}{*}{ Tests empleados } & \multicolumn{2}{|c|}{ Hallazgos } \\
\hline & & Déficit & $\begin{array}{l}\text { Habilidades conserva- } \\
\text { das }\end{array}$ \\
\hline $\begin{array}{l}\text { Hightower y Anderson, } \\
1986\end{array}$ & $\begin{array}{l}\text { Escala Revisada de Memoria Wechsler } \\
\text { (versión de Rusell) }\end{array}$ & $\begin{array}{ll}\text { - } & \text { Memoria general } \\
\text { - } & \text { Aención y concen- } \\
\text { - } & \text { tración } \\
\text { - } & \text { Memoria visual } \\
\end{array}$ & - Memoria verbal \\
\hline Nixon y col., 1987 & $\begin{array}{l}\text { Escala Revisada de Memoria Wechsler } \\
\text { (versión de Rusell) }\end{array}$ & $\begin{array}{ll}\text { - } & \text { Memoria general } \\
\text { - } & \text { Aención y concen- } \\
\text { - } & \text { Meción } \\
\text { - } & \text { Memoria visual } \\
\end{array}$ & - $\quad$ Memoria verbal \\
\hline Eckardt y col., 1995 & $\begin{array}{l}\text { Escala de Memoria Wechsler } \\
\text { Tactual performance test } \\
\text { Test de categorías semánticas }\end{array}$ & & - Memoria \\
\hline Nixon y Bowly,1996 & Aprendizaje de listas verbales & - Memoria de trabajo & \\
\hline Mann y col., 1999 & $\begin{array}{l}\text { Escala de Memoria Wechsler } \\
\text { Batería Halstead Reitan }\end{array}$ & - Memoria de trabajo & \\
\hline Sullivan y col., 2000 & $\begin{array}{l}\text { Brown-Peterson distractor test } \\
\text { Escala de Memoria Wechsler }\end{array}$ & & 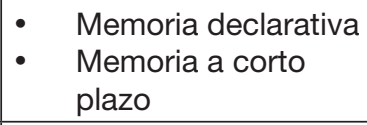 \\
\hline Ambrose y col., 2001 & The delayed alternation task & - $\quad$ Memoria de trabajo & \\
\hline Noël y col, 2001 & Alpha-span task & & - $\quad$ Memoria \\
\hline Sullivan y col., 2002 & Escala de Memoria Wechsler & & $\begin{array}{ll}\text { - } & \text { memoria de trabajo: } \\
\text { - } & \text { bucle articulatorio } \\
\text { - } & \text { agenda visoespacial }\end{array}$ \\
\hline
\end{tabular}

Entre los estudios más completos sobre la memoria destacan los realizados con la versión de Rusell de la Escala Revisada de Memoria Wechsler (Rusell, 1975). Así, varios autores, (Acker, Ron, Lishman y Shaw, 1984; Hightower y Anderson, 1986; Nixon, Kujawski, Parsons y Yohman, 1987), constataron que, al evaluar a sujetos alcohólicos con esta prueba y comparar los resultados obtenidos con un grupo de control, los primeros presentaban deficiencias en los índices de memoria general, atención y concentración, memoria visual y memoria demorada. Sin embargo, los resultados se equiparaban en el índice de memoria verbal. No obstante, a pesar de que las diferencias encontradas entre ambos grupos resultaban, en general, significativas no eran demasiado pronunciadas.

Sin embargo, no existe un consenso con respecto a la alteración de la memoria visual. Algunos estudios (cfr. Yohman y Parsons, 1985) constatan este déficit, pero otros no consiguen replicar estos hallazgos (Brandt, Butters, Ryan y Bayog, 1983; Riege, Tomaszewewski, Lanto y Metter, 1984), o los encuentran solamente durante las primeras semanas de abstinencia (Leber, Jenkins y Parsons, 1981).

Respecto a las investigaciones que tratan de determinar los déficit que se producen en la memoria de trabajo, han sido numerosas las investigaciones que se centran en medir la capacidad para el aprendizaje de listas verbales. Así, son varios los estudios que han hallado alteraciones significativas al evaluar esta capacidad mediante el Rey Auditory Verbal Learning Test (Query y Berger, 1980; Tarquini y Masullo, 1981) o con pruebas similares (Nixon y Bowly,1996).

En esta misma línea, se ha encontrado que estos pacientes presentan dificultades en tareas de aprendizaje de pares asociados (Becker y Jaffe, 1984; Brandt y col., 1983) aunque otros estudios contradicen estos resultados (Yohman y Parsons,1985). En cualquier caso, los déficit variaban en función del tipo de estímulos y de la edad de los sujetos. De esta forma, el rendimiento era más deficitario en aquellas tareas en las que estaban implicados estímulos no verbales (Kapur y Butters, 1977; Leber y col., 1981) y se establecía una relación inversa entre ejecución y edad del individuo (Cermak y Peck, 1982; Ryan y Butters, 1980).

Asimismo, en la investigación de Ambrose, Bowden y Whelan (2001), en la que se utilizó una prueba diseñada específicamente para determinar el nivel de funcionamiento de la memoria de trabajo (The delayed alternation task) se llega a la misma conclusión. Estos autores afirman que este almacén temporal de la memoria se encuentra afectado en sujetos alcohólicos, incluso sin que estén presentes otros déficit cognitivos severos. 
Desde otra perspectiva, al margen de los deterioros de memoria encontrados en los diferentes estudios, es interesante analizar en que medida estas alteraciones se recuperan cuando se mantiene la abstinencia a medio o largo plazo. Algunos autores (cfr. Fein, Bachman, Fisher y Davenport, 1990) afirman que el déficit es amplio y frecuente (hasta dos terceras partes de los alcohólicos) durante los primeros meses tras la desintoxicación y señalan que entre las funciones más dañadas en esos primeros momentos, se encuentra la capacidad para realizar nuevos aprendizajes. Sin embargo, está función, al igual que otras, recupera niveles premórbidos tras un período prolongado de abstinencia.

En este sentido, en la investigación de Mann, Günter, Setter y Ackerman (1999) se observó que de las 12 funciones cognitivas evaluadas, cinco se encontraban alteradas en la primera semana de desintoxicación, pero tan solo una (en concreto, la memoria a corto plazo o memoria de trabajo), continuaba afectada después de la quinta semana de desintoxicación.

A pesar de que existe un relativo consenso sobre la relación entre el consumo de alcohol y la afectación de la memoria, algunos estudios matizan este tipo de relación. Así, en el estudio de Eckardt, Stapleton, Rawlings, Davis y Grodin (1995), realizado con una muestra de 101 alcohólicos jóvenes de entre 18 y 35 años, se encuentra que los resultados, tanto en memoria como en otras funciones cognitivas, se hallan dentro de la normalidad. Sin embargo, sí se muestra una correlación entre los años de consumo y los déficit mnésicos, así como entre el rendimiento en las pruebas y la duración de la abstinencia.

En esta misma línea, en otro estudio realizado con una muestra de 71 alcohólicos recientemente desintoxicados (Sullivan, Rosenbloom y Pfefferbaum, 2000), se evaluó, entre otras funciones, la memoria declarativa y la memoria a corto plazo. Los resultados mostraron que ambas habilidades se encontraban relativamente intactas. Sin embargo, en un estudio similar llevado a cabo con mujeres alcohólicas (Sullivan, Fama, Rosenbloom y Pfefferbaum, 2002) se puso de relieve que tanto el bucle articulatorio como la agenda visoespacial de la memoria de trabajo estaban afectados.

Por último, en otra investigación reciente (Noël y col, 2001) en la que se evaluó una muestra de sujetos alcohólicos tras el período de desintoxicación se concluye que la memoria no se encuentra afectada, aunque sí otras funciones cognitivas. No obstante hay que señalar que la prueba utilizada para la evaluación de la memoria (el Alpha-span task) es demasiado específica y los resultados en esta prueba no se pueden extrapolar al funcionamiento global de la memoria.

En suma, aunque la investigación parece apuntar a una afectación de la memoria causada por el abuso prolongado del alcohol, continúa sin haber unanimidad al respecto. Parece, no obstante que las deficiencias se centran en la memoria declarativa y no en la memoria procedimental. Asimismo, dentro de la memoria declarativa parece ser la memoria de trabajo la más afectada. Este subsistema puede considerarse como un almacén temporal de entrada de información, a partir del cual se constituyen memorias más estables por lo que afecta a su vez a la memoria declarativa episódica a largo plazo. Sin embargo, la memoria semántica, debido a que incluye aprendizajes adquiridos anteriormente a los déficit mnésicos, permanece conservada. Así lo confirma el hecho de que los sujetos alcohólicos ejecuten adecuadamente los tests de inteligencia general, sobre todo las pruebas que evalúan funciones verbales relacionadas con el conocimiento general sobre el mundo, que depende de la memoria semántica (Wilkinson y Carlen, 1980; Knight y Longmore, 1994; Aragón y Miquel, 1995).

\section{DEFICIT EJECUTIVOS}

El córtex prefrontal está considerado como la región cerebral con un desarrollo filogenético y ontogénico más reciente y, por lo tanto, el que mejor refleja nuestra especificidad (Goldman-Rakic,1984). Desde un punto de vista funcional, puede afirmarse que en esta región cerebral se encuentran las funciones cognitivas más complejas y evolucionadas del ser humano, por lo que se le atribuye un papel esencial en actividades tan importantes como la creatividad, la ejecución de actividades complejas, el desarrollo de las operaciones formales del pensamiento, la conducta social, la toma de decisiones o el juicio ético y moral (Pelegrín y Tirapu, 1995). En esta línea, los tipos de déficit que se atribuyen a lesiones del córtex prefrontal incluyen un amplio abanico de alteraciones emocionales, conductuales y cognitivas. Dentro de estas últimas destacan los déficit en las denominadas funciones ejecutivas.

El término "funciones ejecutivas" lo acuñó Lezak $(1982,1987)$ para referirse a un conjunto de capacidades implicadas en la formulación de metas, en la planificación para su logro y en la ejecución de la conducta de un modo eficaz. Ello incluye, entre otros aspectos, la motivación, la conciencia de sí mismo, el pensamiento abstracto o la capacidad para iniciar, proseguir y detener secuencias complejas de conducta de un modo ordenado e integrado.

En los últimos años se ha dedicado una mayor atención a la evaluación específica de los déficit en las funciones ejecutivas en sujetos alcohólicos. Así, varios estudios (Cutting, 1978; Goldstein y Shelley, 1982; Hill, 1980; Jones y Parsons, 1971; Long y McLachlan, 1974; Parsons, 1987; Smith, Burt y Chapman, 1973; Sullivan, Mathalon, Zipursky, Kersteen-Tucker, Knight y Pfferbaum, 1993) han confirmado la presencia de estos défi- 
cit en sujetos alcohólicos. En estos casos aparece el denominado síndrome disejecutivo (Baddeley y Wilson, 1988) caracterizado por dificultades para: a) centrarse en una tarea y finalizarla sin un control externo; b) establecer nuevos repertorios conductuales y utilizar estrategias operativas; c) mantener una conducta flexible y creativa, con una evidente falta de flexibilidad cognitiva; y d) anticipar las consecuencias de su comportamiento, lo que provoca una mayor impulsividad e incapacidad para posponer una respuesta (aspecto crucial en la comprensión de las conductas adictivas).

Un resumen de los resultados de los principales estudios sobre alteraciones de las funciones ejecutivas se presenta en la tabla 2.

TABLA 2. Estudios sobre la afectación de las funciones ejecutivas en sujetos alcohólicos

\begin{tabular}{|c|c|c|}
\hline Autores y año & Tests empleados & Habilidades en las que se constató déficit \\
\hline Sullivan y col., 1993 & & - Capacidad de resolución de problemas \\
\hline Moselhy y col, 2001 & Revisisón de estudios & $\begin{array}{l}\text { - Capacidad para ejecutar tareas psicomotoras complejas } \\
\text { - la capacidad para resolver problemas y manipular concep- } \\
\text { tos abstractos. }\end{array}$ \\
\hline Ihara y col., 2000 & $\begin{array}{l}\text { Amplia batería que incluye } \\
\text { entre otros: el Trail making } \\
\text { test, test de Winsconsin } \\
\text { modificado, test de Stroop, }\end{array}$ & $\begin{array}{l}\text { - Capacidad para resolver problemas cotidianos } \\
\text { - Ejecución de tareas visoespaciales } \\
\text { - Flexibilidad mental } \\
\text { - La inhibición de respuestas automáticas }\end{array}$ \\
\hline Nöel y col. 2001 & $\begin{array}{l}\text { Torre de Londres, } \\
\text { Test de flexibilidad, } \\
\text { Test de Stroop }\end{array}$ & $\begin{array}{l}\text { - Capacidad de flexibilidad para planificar } \\
\text { - Capacidad para inhibir respuestas }\end{array}$ \\
\hline Sullivan y col. 2000 & $\begin{array}{l}\text { Test de Winsconsin } \\
\text { (presentación verbal y no } \\
\text { verbal) }\end{array}$ & - Habilidades verbales y no verbales \\
\hline Ratti y col., 2002 & Test de Winsconsin & - Flexibilidad mental \\
\hline Corral y col., 2002 & Test de Winsconsin & - Flexibilidad mental \\
\hline
\end{tabular}

En la amplia revisión llevada a cabo por Moselhy, Georgiou y Kahn, (2001) sobre los estudios que se centran en la afectación frontal de los alcohólicos, se concluye que presentan una afectación neuropsicológica en las funciones atribuidas al lóbulo frontal. Destaca, además, que este déficit es especialmente marcado en la capacidad para ejecutar tareas psicomotoras complejas y en la capacidad para resolver problemas y manipular conceptos abstractos.

De la misma forma, en el estudio de Ihara, Berrios y London, (2000) se llevó a cabo una evaluación neuropsicológica de un grupo de diecisiete alcohólicos no afectados por amnesia. Los resultados mostraron que, comparados con el grupo de control, los alcohólicos presentaban disfunciones ejecutivas. Esta disfunción afectaba tanto a aspectos globales (como la capacidad para resolver problemas cotidianos), como a aspectos más específicos (como la ejecución de tareas visoespaciales, la flexibilidad mental y la inhibición de respuestas automáticas).

En este último trabajo resulta de particular interés el análisis detallado de los resultados en las diferentes pruebas. En concreto de esta forma se obtienen cuatro perfiles diferenciados: 1) los que presentan un síndrome disejecutivo con memoria e inteligencia preservada, 2) los que presentan un síndrome disejecutivo asociado a un déficit de memoria, pero con la inteligencia pre- servada, 3) los que manifiestan un deterioro cognitivo global y 4) los que no presentan déficit cognitivo alguno. Aproximadamente dos tercios de los pacientes se situaban en los dos primeros grupos, presentando, por tanto, una alteración en las funciones ejecutivas. Estos datos muestran la heterogeneidad de los sujetos alcohólicos y la necesidad de diseñar estudios que pongan en relación variables significativas con los diferentes perfiles neuropsicológicos encontrados.

En la misma línea, en otro estudio neuropsicológico con treinta sujetos alcohólicos no afectados por amnesia (Nöel y col., 2001) se obtiene una diferencia significativa entre el rendimiento en pruebas que evalúan funciones ejecutivas y aquellas que valoran otras funciones cognitivas (que no se hallaban afectadas). En la batería de evaluación se incluyeron diferentes pruebas que evalúan funciones ejecutivas siguiendo el modelo del sistema Atencional Supervisor de Shallice (1988) y el modelo de Memoria de Trabajo de Baddeley (1974), entre las que se incluían la torre de Londres, test de flexibilidad, y el Test de colores y palabras Stroop. Los resultados obtenidos señalan una alteración en la capacidad de flexibilidad para planificar (que resultaba en una dificultad para corregir sus propios errores y reconducir la respuesta) y un déficit en la capacidad para inhibir respuestas lo que diferentes trabajos relacionan con una afectación del lóbulo frontal como estructura y del funcionamiento ejecutivo como función. 
Asimismo, otras investigaciones (Sullivan y col., 2000; Ratti, Bo, Giardini y Soragna, 2002; Corral, Rodríguez y Cadaveira, 2002) en las que se evalúan las funciones ejecutivas en sujetos alcohólicos mediante el Winsconsin Card Sorting Test (Heaton, Chelune, Talley, Kay y Curtis, 1993) corroboran estos resultados.

La presencia de una disfunción de los lóbulos frontales tiene importantes implicaciones. Por un lado, desde una perspectiva terapéutica, estos déficit pueden afectar al éxito de los diferentes programas de tratamiento. Por ello, puede ser necesario modificar dichos programas para que se adapten a las capacidades cognitivas de los pacientes (McCrady y Smith, 1986). Por otro lado, la presencia de déficit neuropsicológicos puede tener un importante valor predictivo para la aparición de trastornos neurológicos propios del alcoholismo. Así, en el estudio de Tuck y Jackson (1991), con 641 alcohólicos, se constató que la existencia de alteraciones en la memoria a corto plazo y en los lóbulos frontales precedía, en un plazo de hasta 10 años, a los trastornos neurológicos relacionados con el alcohol.

Algunos autores han señalado que el déficit de las funciones ejecutivas se puede entender como un factor predisponente para padecer problemas de consumo de alcohol o como una mayor vulnerabilidad a la aparición de consecuencias adversas con un patrón normal de consumo. Un resumen de algunos estudios que apoyan está hipótesis se presenta en la tabla 3.
En esta línea de investigación destaca el estudio de Giancola, Zeichner, Yarnell y Dickson (1996), en el que se evaluaba a 79 bebedores sociales no alcohólicos con edades comprendidas entre los 17 y 30 años. Por un lado, se evaluaron las funciones ejecutivas mediante el (SMMT). Por otro lado, se evaluaban las consecuencias negativas del consumo de alcohol en cinco áreas (médica, intrapersonal, interpersonal, responsabilidad social y control de impulsos). Los resultados reflejaban una correlación entre la ejecución deficitaria en estas pruebas y el mayor número de consecuencias negativas en todas las áreas, excepto en la referida a las consecuencias médicas. Estos resultados ponen de manifiesto la relación existente entre un déficit en las funciones ejecutivas y la presencia de mayores consecuencias negativas del consumo de alcohol.

Por otro lado, se ha hipotetizado que una hipofunción del córtex frontal podría ser un factor predisponente de la dependencia acohólica. Sin embargo, existen problemas metodológicos importantes en los estudios llevados a cabo, ya que la mayoría de ellos han realizado con sujetos en los que el consumo de alcohol estaba ya instaurado. Por ello resulta difícil determinar si el déficit en las funciones ejecutivas es un desencadenante o una consecuencia del abuso.

Un intento de solucionar estos problemas metodológicos son los estudios con hijos de alcohólicos, ya que la probabilidad de desarrollar alcoholismo en esta población es hasta cinco veces superior con respecto

TABLA 3. Estudio sobre alteraciones de las funciones ejecutivas en sujetos predispuestos a desarrollar abuso y dependencia a sustancias.

\begin{tabular}{|l|l|l|l|}
\hline \multicolumn{1}{|c|}{ Estudio } & \multicolumn{1}{|c|}{ Tipo de muestra } & $\begin{array}{l}\text { Test empleados o funciones } \\
\text { evaluadas }\end{array}$ & \multicolumn{1}{c|}{ Resultados } \\
\hline Giancola y col., 1996 & 79 bebedores sociales & $\begin{array}{l}\text { Test de Winsconsin, el } \\
\text { Conditional Associative } \\
\text { Learning Test y el Sequential } \\
\text { Matching Memory Test }\end{array}$ & $\begin{array}{l}\text { Correlación entre la ejecución } \\
\text { deficitaria en estas pruebas } \\
\text { y el mayor número de } \\
\text { consecuencias negativas del } \\
\text { consumo }\end{array}$ \\
\hline Corral y col., 1999 & $\begin{array}{l}\text { 102 sujetos de 7 a 15 } \\
\text { años de edad que fueron } \\
\text { agrupados en función de } \\
\text { la densidad familiar de } \\
\text { alcoholismo }\end{array}$ & Atención y funciones frontales & $\begin{array}{l}\text { Los sujetos con mayor } \\
\text { densidad de alcoholismo } \\
\text { ejecutaban peor las pruebas }\end{array}$ \\
\hline Tapert y col., 2002 & $\begin{array}{l}66 \text { jóvenes considerados de } \\
\text { alto riesgo }\end{array}$ & procesos atencionales & $\begin{array}{l}\text { La capacidad atencional } \\
\text { predecía posteriores } \\
\text { problemas de consumo }\end{array}$ \\
\hline Giancola y Parker, 2001 & 187 niños & $\begin{array}{l}\text { Los déficit ejecutivos predicen } \\
\text { consumo de tóxico en la } \\
\text { adolescencia }\end{array}$ \\
\hline
\end{tabular}

a la población normal (Cloninger, Bohman y Siguardson, 1981) y, en ellos no interfieren los efectos tóxicos del consumo. En este sentido, varios estudios han señalado la presencia de déficit neuropsicológicos en hijos de alcohólicos (Sher,1991; Díaz, Polo, Escera, Sánchez-
Turet, y Grau, 1999. Además, algunas investigaciones confirman que el grado de déficit está en función de la densidad familiar. Esto es, las alteraciones resultan más graves en los hijos que presentan antecedentes de alcoholismo en varias generaciones, con respecto a los 
que tienen una sola cohorte afectada (Corral, Rodríguez y Cadaveira,1999).

En esta línea, en un estudio de revisión de Giancola y Moss (1998) se llega a la conclusión de que tanto los alcohólicos como los niños con alto riesgo para desarrollar alcoholismo presentan una disfunción en el córtex prefrontal. Desde un punto de vista neurobiológico esto corresponde a la incapacidad del córtex prefrontal para regular e inhibir mensajes estereotipados del incentivo del consumo de drogas que provienen del núcleo estriado. Otros estudios llevados a cabo por este mismo grupo (Giancola y Parker, 2001) constatan la relación existente entre déficit ejecutivos en la infancia y consumo de tóxicos en la adolescencia, por lo que estos datos cobran una gran importancia desde una perspectiva preventiva y plantean la necesidad de fomentar el desarrollo de las funciones ejecutivas en niños que pertenecen a grupos de alto riesgo.

Lo mismo ocurre en el estudio de Tapert, Baratta, Abrantes y Brown, (2002), que trata de determinar el papel desencadenante de los déficit ejecutivos (más concretamente, procesos atencionales) en los problemas de consumo de drogas. Para ello se evalúa a 66 jóvenes considerados de alto riesgo y se realiza un seguimiento de ocho años. Los resultados indican que las puntuaciones obtenidas en la evaluación neuropsicológica de la capacidad atencional predecían el uso de sustancias y los síntomas de dependencia 8 años más tarde. Por tanto, parece que el déficit atencional, incluso sin diagnóstico de trastorno por déficit de atención o hiperactividad, actuaría como un factor de vulnerabilidad para desarrollar problemas de consumo de alcohol u otros tóxicos. Insisten asimismo en la importancia de incluir estos conocimientos en las intervenciones de carácter preventivo. Un ejemplo en este sentido es el "Programa Alfil" llevado a cabo en nuestro país por Díaz Hurtado, Gual, Serrano, Costa, Ferri y Grau (2001).

No obstante, hay que tener en cuenta que las disfunciones del lóbulo frontal pueden ser a la vez causa y consecuencia del alcoholismo. Por lo que se necesita un mayor número de estudios que clarifiquen este aspecto.

\section{CONCLUSIONES}

En este texto se ha llevado a cabo un análisis de las principales investigaciones que estudian la relación entre el alcoholismo, las alteraciones de la memoria y los déficit ejecutivos.

Por lo que se refiere a la memoria, los resultados de los diferentes estudios ponen de manifiesto, por una parte, la existencia de un deterioro en la memoria a corto plazo y, por otra, la preservación de la memoria procedimental. No obstante, existen discrepancias en cuanto a si el déficit es mayor en el componente verbal o visual de la memoria de trabajo.
Por otro lado, hay que señalar que, aun cuando la memoria incluye tres procesos diferenciados, (adquisición, almacenamiento y recuperación), la mayoría de los estudios se refieren exclusivamente al de adquisición. Por esto, se puede afirmar que la capacidad para adquirir nueva información (aprendizaje) se encuentra afectada pero se desconoce aún si los procesos de almacenamiento y recuperación están asimismo afectados.

Otro aspecto sobre el que tampoco existe un consenso es el grado en que los déficit observados pueden recuperarse. En este sentido, es importante conocer tanto el aspecto temporal, esto es, en que período de tiempo se recupera la capacidad, como el carácter parcial o total de la recuperación. Asimismo existen discrepancias en cuanto a la recuperación diferencial de los diferentes tipos de memoria.

En definitiva, los resultados obtenidos son heterogéneos debido, probablemente, a la falta de distinción entre los diferentes subsistemas de memoria y de los procesos implicados en las capacidades mnésicas. Además, en las futuras investigaciones se deben controlar también otras variables de tipo sociodemográfico, como la edad (que parece tener una gran influencia en el rendimiento cognitivo) o el nivel cultural (que cuanto más elevado sea, mejor será también la ejecución en las pruebas). Tampoco se deben olvidar los aspectos relacionados con el consumo de alcohol (número de años de consumo, cantidad consumida, duración del período de abstinencia, etc.). Por último, es importante precisar el tipo de pruebas de evaluación utilizadas y concretar, a la luz de los conocimientos existentes sobre la memoria, qué funciones concretas de la memoria evalúan.

Por lo que se refiere a las funciones ejecutivas, la totalidad de los estudios revisados señalan una afectación de estas funciones que se encuentran implicadas en la resolución de situaciones novedosas y que incluyen aspectos tan diversos como la planificación, la abstracción, la inhibición de respuestas irrelevantes, la flexibilidad mental, la generación de conceptos, los ensayos mentales y el reconocimiento del logro.

No obstante, también se observan limitaciones importantes. Probablemente éstas proceden en parte de la propia definición del concepto que resulta ser descriptivo, pero no ayuda a comprender la etiología funcional de las actividades cognitivas que las sustentan. Por ello, existen múltiples descripciones que no acaban de definir los procesos responsables de una conducta ejecutiva. De hecho, algunos autores señalan que este término se ha convertido en un "paraguas conceptual" y que se precisa una integración de modelos que ayude a comprender los procesos ejecutivos, ya que, posiblemente, no se trate de un sistema unitario, sino más bien, de un sistema supramodal de 
procesamiento múltiple (Tirapu, Muñoz-Cespedes y Pelegrín, 2002).

En referencia a los modelos de deterioro que tratan de explicar el funcionamiento cognitivo en sujetos alcohólicos, hay que señalar que el déficit de las funciones ejecutivas y de la memoria de trabajo apoyan la hipótesis de la vulnerabilidad del lóbulo frontal. En cualquier caso, es importante señalar que estas hipótesis, en ningún caso son incompatibles, sino que podrían mostrar diferentes perspectivas del perfil de deterioro.

Otro aspecto relevante es la afectación de las funciones ejecutivas de forma previa al consumo de alcohol. Ello se ha considerado como un factor predisponente o de vulnerabilidad a padecer problemas derivados de dicho consumo. Algunos trabajos llevados a cabo con hijos de alcohólicos, o los estudios longitudinales sobre déficit neuropsicológicos en niños, parecen encontrar una relación entre los déficit ejecutivos y la vulnerabilidad al alcoholismo. Esta hipótesis no debe conducir a plantear una disfunción del córtex prefrontal como factor "heredado" versus "aprendizaje" en relación con la predisposición, sino más bien, a una interpretación multicausal donde una vulnerabilidad genética interactúa con un ambiente empobrecido y no permite que se den las conexiones neurales necesarias para un correcto funcionamiento cerebral.

\section{REFERENCIAS BIBLIOGRAFICAS}

Acker W, Ron MA, Lishman WA y Shaw GK (1984): A multivariate analysis of psychological, clinical and CT scanning measures in detoxified chronic alcoholics. British Journal of Adiction, 79: 293-301.

Ambrose ML, Bowden SC y Whelan G (2001): Working memory impairments in alcohol-dependent participants without clinical amnesia. Alcoholism: Clinical and Experimental Research, 25:185-191.

Aragón CM y Miquel M (1995): Alcoholismo. En A Belloch, B Sandín, F Ramos (Eds.): Manual de Psicopatología. Madrid. Mc Graw Hill.

Baddeley AD y Hitch GJ (1974): Working memory. En Bower (Eds.): Recent advances in learning and motivation, vol VIII, New York. Academic Press.

Baddeley AD y Wilson BB (1988): Frontal amnesia and dysexecutive syndrome. Brain and Cognition, 7: 212-30.

Becker JT y Jaffe JH (1984): Impaired memory for treatment relevant information in inpatient men alcoholics. Journal of Studies on Alcohol,45: 339-343.

Bertera JH y Parsons OA (1978): Impaired visual search in alcoholics. Alcoholism: Clinical and Experimental Research, 2: 9-14.

Brandt J, Butters N, Ryan C y Bayog R (1983): Cognitive loss and recovery in long-term alcohol abusers. Archives of General Psychiatry, 40: 435-442.
Caballero Martínez L (2000): Adicción a la cocaína: de la neurobiología a la clínica humana. JANO. Psiquiatría y humanidades. Vol II, 1:5-9.

Cermak LS y Peck E (1982): Continuum versus premature aging theories of chronic alcoholism. Alcoholism: Clinical and Experimental Research,6: 89-95.

Cloninger CR, Bohman M y Siguardson S (1981): Inheritance of alcohol abuse: Cross fostering analysis of adopted men. Archives of General Psychiatry, 38: 861-867.

Corral MM, Rodriguez S y Cadaveira F (1999): Neuropsychological characteristics in children of alcoholics: familial density. Journal of Studies on Alcohol,60: 509-13.

Corral MM, Rodríguez, S y Cadaveira F (2002): Perfil neuropsicológico de alcohólicos con alta densidad familiar de alcoholismo tras abstinencia prolongada: hallazgos preliminares. Revista Española de drogodependencias, 27: 148-158.

Cutting J (1978): Specific psychological deficits in alcoholism. British Journal of Psychiatry, 133:119-122.

Deckel AW, Hesselbrock V y Basuer L (1996): Antisocial personality disorder, childhood delinquency, and frontal brain functioning: EEG and neuropsychological findings. Journal of Clinical Psychology,52: 639-50.

Díaz, RM, Gual, A, Serrano, I, Costa, S, Ferri, MJ y Grau, C (2001): Programa ALFIL: evaluación de marcadores de riesgo e intervención preventiva en hijos de alcohólicos. Adicciones, 13: 39-49.

Díaz R, Polo MD, Escera C, Sánchez-Turet M, y Grau C (1999): Marcadores neurogenéticos de riesgo para el alcoholismo. En M. Sanchez-Turet (Ed.): Enfermedades y problemas relacionados con el alcohol.Barcelona. Ediciones ESPAX.

Eckardt MJ, Stapleton JM, Rawlings RR, Davis EZ y Grodin DM (1995): Neuropsychological functioning in detoxified alcoholics between 18 and 35 years of age. American Journal of Psychiatry, 152:53-59.

Fassino S, Piero A, Daga GA, Leombruni P, Mortara P y Rovera GG (2002): Attentional biases and frontal functioning in anorexia nervosa. International Journal of Eating Disorders, 31:274-83.

Fein G, Bachman L, Fisher S y Davenport L (1990): Cognitive impairments in abstinent alcoholics. Addiction Medicine, 152: 531-537.

Fernández-Espejo (2002): Bases neurobiológicas de la drogadicción. Revista de Neurología,34: 659-64.

Giancola PR, Zeichner A, Yarnell JE y Dickson KE (1996): Relation between executive cognitive functioning and the adverse consequences of alcohol use in social drinkers. Alcoholism: Clinical and Experimental Research, 20:1094-1098.

Giancola PR y Moss HB (1998): Executive cognitive functioning in alcohol use disorders. Recent developments in alcoholism,vol.14: The consecuences of alcoholism, New York. Plenun Press.

Giancola PR y Parker AM (2001): A six year prospective study of pathways toward drug use in adolescent boys 
with and without a family history of a substance use disorder. Journal of Studies on Alcohol ,62:166-78.

Goldman-Rakic PS (1984): The frontal lobes: uncharted provinces of the brain. TINS, 7:425-9

Goldstein G y Shelly C,(1982): Neuropsychological investigation of brain lesion localisation in alcoholism. En H Begleiter y B Kissin (Eds.): International Symposium on biological Research in alcoholism, New York. Plenum Press.

Gómez Bosque P (1992): Memoria y cerebro. En D Barcia Salorio: Trastornos de la memoria. Barcelona. MCR.

Graham FK y Kendall BS, (1960): Memory-For-Designs-Test: Revised General Manual(Monograph). Perceptual and Motor Skills, 11:147-188.

Head D, Bolton D y Hymas N (1989): Deficits in cognitive shifting ability in patients with OCD. Biological Psychiatry, 25: 929-37.

Heaton RK, Chelune CJ, Talley JL, Kay GG y Curtiss G (1993): Wisconsin Card Shorting Test. Odessa FL: Psychological Assessment Resources. (Edición Española. TEA.1997)

Hightower MG y Anderson RP (1986): Memory evaluation of alcoholics with Russell's revised wechsler memory Scale. Journal of Clinical Psychology,42: 1000-1005.

Hill SY (1980): Comprehensive assessment of brain dysfunction in alcoholic individuals. Acta Psychiatrica Scandinavica, 62: 57-75.

Ihara H, Berrios GE y London M (2000): Group and case study of the dysexecutive syndrome in alcoholism without amnesia. Journal of Neurology, Neurosurgery and Psychiatry, 68: 731-737.

Iruarrizaga I, Miguel-Tobal JJ, Cano A (2001): Alteraciones neuropsicológicas del alcoholismo crónico. Un apoyo a la hipótesis del continuo. Psicothema, 13: 571-580.

Jones B y Parsons OA (1971): Impaired abstracting ability in chronic alcoholics. Archives of General Psychiatry,24: 71-75.

Kapur N y Butters N (1977): Visuoperceptive deficts in long term alcoholics and alcoholics with Koooorsakoff's psychosis. Journal of Studies on Alcohol,38: 2025-2035.

Knight RG y Longmore BE (1994): Clinical Neuropsychology of Alcoholism. LEA. Hove.

Krabbendam L, Visser PJ, Derix MM, Verhey F, Hofman P, Verhoe W, Tuinier S y Jolles J (2000): Normal cognitive performance in patients with chronic alcoholism in contrast to patients with korsakoff's syndrome. Journal of Neuropsychiatry and Clinical Neuroscience, 12: 44-50.

Leber WR, Jenkins RL y Parsons OA (1981): Recovery of visual spatial learning and memory in chronic alcoholics. Journal of Clinical Psychology,37, 192-197.

Lezak MD (1982): The problem of assesing executive functions. International Journal of Psychology,17: 281-97.

Lezak MD (1987): Relationship between personality disorders, social disturbances and physical disability following traumatic brain injury. Journal of Head Trauma Rehabilitation, 2: 57-69.

Long JA y McLachlan JF (1974): Abstract reasonig and perceptual motor efficiency in alcoholics. Impairmente and reversibility. Quaterly Journal of Studies on Alcohol, 35: 1220-29.

Mann K, Günter A, Setter F y Ackerman K (1999): Rapid recovery from cognitive deficits in abstinent alcoholics: a controlled test-retest study. Alcohol and alcoholism, 34: 567-574.

McCrady BS y Smith DE (1986): Implications of cognitive impairment for the treatment of alcoholism. Alcoholism Clinical and Experimental Research, 10:145-9.

Moshely HF, Georgiou G y Kahn A (2001): Frontal lobe changes in alcoholism: a review of the literature. Alcohol and Alcoholism, 36: 357-68.

Nixon SJ y Bowlby D (1996): Evidence of alcohol-related efficiency deficits in an episodic learning task. Alcoholism Clinical and Experimental Research, 20: 21-4.

Nixon SJ, Kujawski A, Parsons OA y Yohman JR (1987): Semantic (verbal) and figural memory impairment in alcoholics. Journal of Clinical and Experimental Neuropsychology, 9: 311-322.

Nöel X, Van der Linden M, Schmidt N, Sferraza R, Hanak C, Le Bon O, De Mol J, Kornreich Ch, Pelc I y Verbank P (2001): Supervisory Attentional System in nonamnesic alcoholic men. Archives of General Psychiatry, 58: 11521158.

Oscar-Berman M y Ellis RJ (1987): Cognitive deficits related to memory impairments in alcoholism. En M Galanter (Eds.): Recent developments in alcoholism. New York. Plenun Press.

Parsons OA (1987): Neuropsychological consequences of alcohol abuse: many questions, some answers. En O Parsons, N Butters, P Nathan, (Eds.): Neuropsychology of Alcoholism: Implications for Diagnoses and Treatment, , 153-173. New York. Guildford Press.

Pelegrín C y Tirapu J (1995): Neuropsiquiatría del daño prefrontal traumático. Monografías de Psiquiatría, 7:11-21.

Query WT y Berger RA (1980): AVLT memory scores as a function of age among general medical, neurologic and alcoholic patients. Journal of Clinical Psychology, 36: 1009-1012

Ratti MT, Bo P, Giardini A y Soragna D, (2002): Chronic alcoholism and the frontal lobe: which executive functions are impaired? Acta Neurologica Scandinavica, 105, 276.

Ratti MT, Soragna D, Sibilla L, Giardini A, Albergati A, Savoldi F y Bo P: Cognitive impairment and cerebral atrophy in "heavy drinkers". Pro Neuropsychopharmacol biol Psychiatry, 23: 243-58.

Riege WH, Tomaszewewski R, Lanto A y Metter EJ (1984): Age and alcoholism: Independent memory decrements. Alcoholism: Clinical and experimental Research, 8: 4247.

Rusell EW (1975): A multiple scoring method for the assessment of complex memory functions. Journal of Consulting and Clinical Psychology, 43: 800-809.

Ryan C y Butters N, (1980): Further evidence for a continuum of impairment encompassing male alcoholic korsakoff patients and chronic alcoholic men. Alcoholism: Clinical and Experimental Research,4: 190-198. 
Shallice T (1988): From neuropsychology to mental structure. Cambridge: Cambridge University Press.

Sher KJ (1991): Children of alcoholics. Acritical appraisal of theory and research. Chicago and London. University of Chicago Press.

Smith MA, Burt DW y Chapman RF (1973): Intelligence and brain damage in alcoholics. A study in patients of middle and upper social class. Quaterly Journal of Studies on Alcohol 34: 414-422.

Squire LR (1975): Short term memory as a biological entity. En: Short term memory. New York Academic Press.

Squire LR y Butters N.(1992): Neuropsychology of memory. $\left(2^{a}\right.$ ed) New York. Guildford Press.

Sullivan EV, Fama R, Rosenbloom MJ y Pfefferbaum A (2002): A profile of neuropsychological deficits in alcoholic women. Neuropsychology, 16: 74-83.

Sullivan EV, Mathalon DH, Zipursky RB, Kersteen-Tucker Z, Knight RT y Pfefferbaum A (1993): Factors of the Winsconsin Card Sorting Test as measures of frontal lobe function in schizoprenia and in chronic alcoholism. Psychiatry Research, 46:175-199.

Sullivan EV, Rosenbloom MJ y Pfefferbaum A (2000): Pattern of motor and cognitive deficits in detoxified alcoholic men. Alcoholism: Clinical and Experimental Research, 24: 611-621.

Tapert SF, Baratta MV, Abrantes AM y Brown SA (2002): Attention dysfunction predicts substance involvement in community youths. Journal of American Academic Children and Adolescent Psychiatry,41: 680-686.

Tarquini D y Masullo C (1981): Cognitive impairments and chronic alcohol abuse: A neuropsychological study. Drug and Alcohol Dependence, 8: 103-109.

Tarter RE (1975): Brain damage associated with chronic alcoholics, Disease of Nervous System,36: 185-87.

Tarter RE (1976): Neuropsychological investigations of alcoholism. En: G Goldstein, C Neuringer (Eds.): Empirical Studies of alcoholism: 231-256. Cambridge, Mass.Ballinger.
Tirapu Ustárroz J, Casi Arbonies A y Ugarteburu I, (1997): Aplicación de las técnicas de modificación de conducta al daño cerebral. En C Pelegrín, JM Muñoz Céspedes, JI Quemada (Eds.): Neuropsiquiatría del daño cerebral traumático. Aspectos clínicos y terapéuticos. Barcelona. Prous Science.

Tirapu Ustárroz J, Muñoz Céspedes JM y Pelegrín Valero C (2002): Funciones ejecutivas: necesidad de una integración conceptual. Revista de Neurología, 34: 67385.

Tuck RR, Jackson M, (1991): Social, neurological and cognitive disorders in alcoholics. Medical Journal of Australia, 155: 225-9.

Tulving E, (1972): Episodic and semantic memory. En E Tulving y W Donaldson (Eds.): Organization of memory. Nueva York: Academic Press.

Tulving E, (1993): Varieties of consciusness and levels of awareness in memory. En A Baddeley y $L$ Weiskrantz (Eds.): Attention: Selection, awareness and control. Atribute to Donald Broadbent. Oxford: Oxford University Press.

Vallar G: Neuropsychological disorders of memory. En G Denes, L Pizzamiglio (Eds.): Handbook of clinical and experimental neuropsychology. UK. Psychology Press. Taylor \& Francis.

Walker K (1976): Memory. En: Clinical methods. The history of the physical and laboratoty examination. Butterworth, Boston.

Wilkinson DA, Carlen PL (1980): Chronic organic brain Syndromes associated with alcoholism: Neuropsychological and other aspects. En Research Advances in alcohol and Drug Problems. Vol.6: 107-145. Plenun Press.

Yohman JR, Parsons OA, (1985): intact verbal pairedassociate learning in alcoholics. Journal of Clinical Psychology, 41: 844-851.

Zola S (1997): Amnesia: neuroanatomic and clinical aspects. En: T Feimberg, MJ Farah (Eds.): Behavioral neurologic and neuropsicologic. New York. Mc Graw Hill. 
